We appreciate the response to this publication feature and welcome all contributions. Contributions may be sent to Phil Oshel, our Technical Editor, at his e-mail address: peoshel@facstaff.wisc.edu. Phil can also be reached at 1-(608) $833-2885$

\section{A Note on lodine and Vacuum Pumps}

Since iodine is a reactive gas, an activated carbon trap is required between the mechanical pump and the turbopump. When I was at the University of Alabama at Birmingham, students on two occasions left the iodine gun valves open. They destroyed the mechanical pump springs and rendered it useless. I found out that we were not the only ones that this happened to. GATAN fixed a bunch of mechanical pumps under warranty and came up with a fix: a carbon trap with a sight glass on the mechanical pump side. Inside the tube where it could be seen, there was a silver strip that would change to black when the trap got saturated. The trap was regenerated by replacing the charge of activated carbon, otherwise iodine would get into the mechanical pump. I would worry that copper pipe used for an exhaust line might react similarly to iodine as the silver. The iodine does come out the exhaust of the mechanical pump.

I thought that the fix was rather expensive at the time, but it did work. A cheaper way to do this would be to get a zeolite trap and replace the zeolite with activated carbon from a pet store that sells aquarium supplies.

I should add that even though the iodine guns were left open twice, the turbo pump was in pristine condition afterward. It was the mechanical pump on the inside, mostly at the exhaust that was saturated with the iodine, and where the mess was.

Scott D. Walck, PPG Industries, Inc. Walck@PPG.com

\section{A Simple Way to Eliminate Frost Build-up on Cryo-SEM Samples}

In the event of frost contamination of a sample, here is a cheap trick that I use to sublimate it off the surface once it is in the SEM: Just hook up a bottle of dry nitrogen gas to the air input of the SEM via a regulator set to about 3 to 5 psi. Vent to atmosphere, do not open chamber door, and then immediately pump back down. All the while the cryostage is kept at usual maximum cold temperature, about $-120^{\circ} \mathrm{C}$ in my case. The gas imparts just enough thermal energy to the surface frost to sublimate it off, but not enough nor long enough to sublimate the sample itself. It may not even be necessary to vent the SEM all the way to room pressure before pumping back downexperiment and see what works.

The big advantage is this only takes 4 minutes for a complete defrost cycle. A disadvantage is that the beam must be turned off, so the process can't be watched, but this is not a problem. I prefer not to defrost by heating up the stage, the sample stub, and the entire mass of the sample just to get some heat to the surface frost, as it takes so long to do that, plus cool down again, and it runs the risk of overheating and drying out or etching the surface layers of the sample. Also, with my model of cryo-system (Emitech $K-1150$, little sibling to the $K-1250$ ) if I should need to

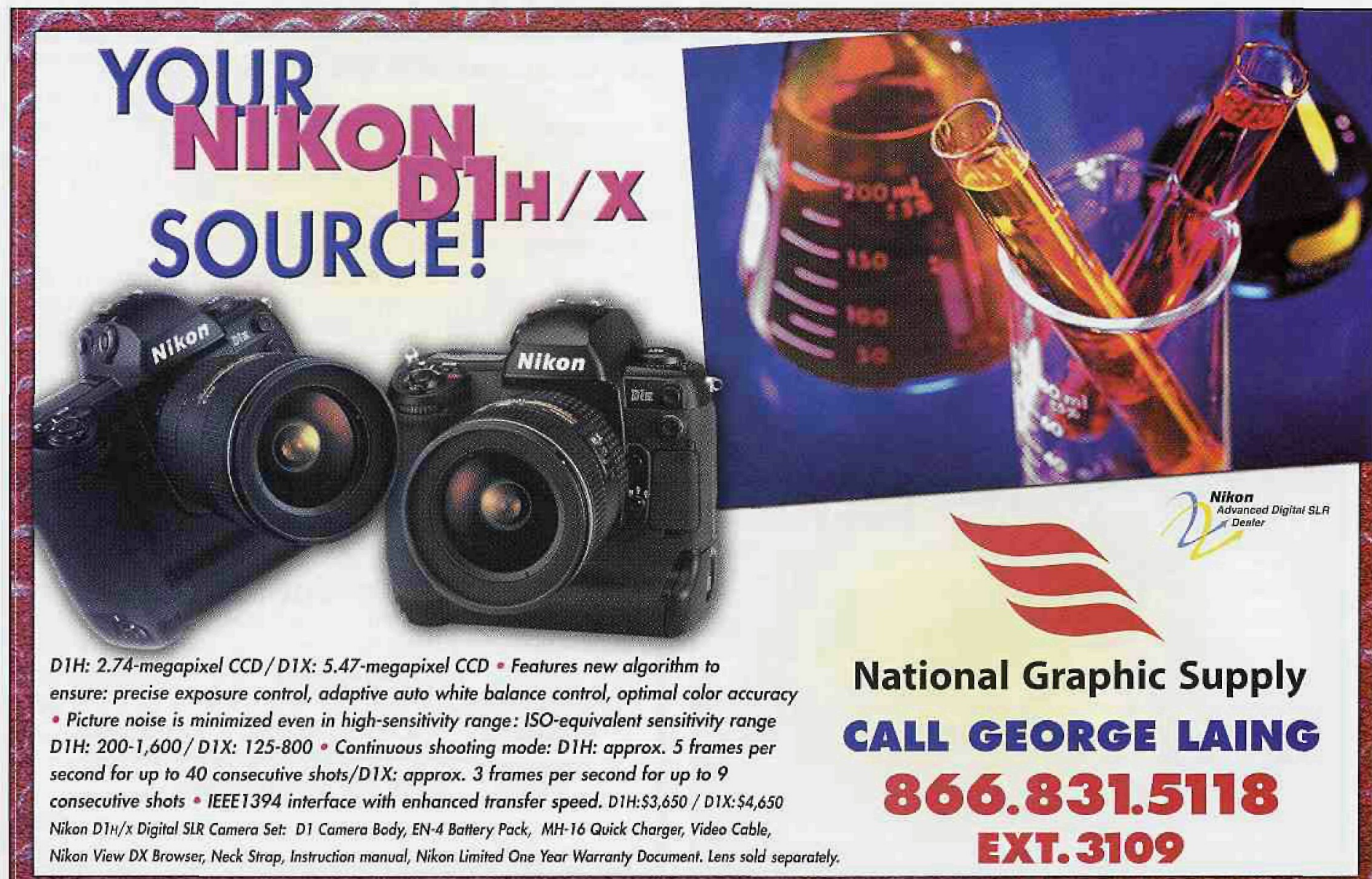

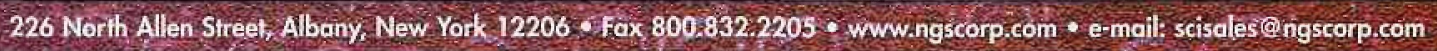


resputter after a defrost, I just pull the sample back into the sputter coater \& airlock transfer unit, which is mounted directly onto the SEM chamber, so there is no further transfer in air involved.

Again, it is rare that I have to defrost now, on my current system. Before, on my old SEM, I had no cryo-prep unit at all, just a cold stage in the SEM, and I had to do transfers in air without any frost shroud protection (which my current Emitech 1150 has), so I had to do this nitrogen gas defrost more often back then.

The micrographs in this reference show an example of the results obtained: "Low-temperature low-voltage scanning electron microscopy of uncoated frozen biological materials: A simple alternative." 1996. Microscopy \& Microanalysis. pp 918-19. (Minneapolis MSA meeting).

Gib Ahlstrand, University of Minnesota ahlst007@tc.umn.edu

\section{Mousescope ${ }^{\mathrm{TM}}$}

Those people who have recently bought a new computer have likely enjoyed the new optical mouse that came with it, especially the cordless variety. But how many have wondered exactly how it works? This isn't the old UNIX optical mouse that required a special mouse pad with a reflective surface and a grid of lines on it. These new mice work on most any surface.

Anyone sufficiently curious to vivisect such a mouse has discovered a cheap plastic lens, a LED light source, and a digital camera (aka "optical sensor," which sounds better to the Marketing types).

Well.

\section{-NORTHERN \\ LIMITED TIME OFFER

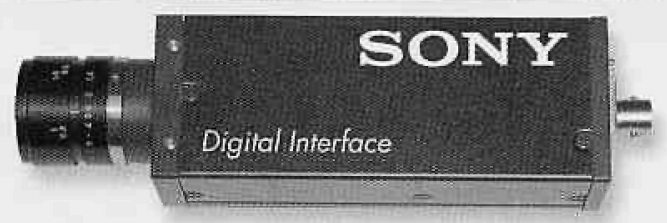 \\ Sony Empix Digital Imaging System \\ Northern Eclipse or Northern Elite 6.0 PLUS Sony Digital Camera with Firewire ${ }^{\mathrm{TM}}$}

Get the most powerful imaging software and a high quality Sony Digital CCD camera for one low price. Includes 3 levels of automation, De-convolution, 3-D Reconstruction, Adaptive Threshold, AVI support, and hundreds of other processing and analysis functions, plus a Sony Digital Mega Pixel Camera. Sony XCD $X 700$ camera, Northern Elite software, card and cable for only $\$ 3695.00$ U.S. Contact Empix Imaging Inc. at: www.empix.com

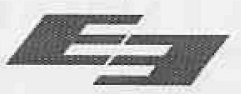

EMP IX
We at the Piltdown Research Institute are just such curious types, and among other things, we found that the camera isn't too bad. It operates at around $18 \times 10^{6}$ instructions per second and takes 800 dpi images at a rate of 1,500 to 2,500 images per second. Some of these mice have two cameras (a different genus no doubt, unless computers have developed homeobox mutants). The signal processor in such a mouse then uses the image to determine where the mouse is, and how much it's moved in what direction, sending the cursor flying across the screen to zap yet another bad guy ... oop, sorry, click on the FFT function. We are scientists here.

Well, again. And two cameras no less.

The next steps in our foray into microelectronics surgery were obvious. First, add a second LED light source to increase the available light on the surface upon which the mouse sits. Then, replace the cheap, plastic lens with a quality glass lens, cheaply acquired (note to Assistant Professors: lock up your compound microscopes when you go on holiday). Next, as we were using a two-camera mouse, slightly reposition the camera chips so that they are looking at the same spot from slightly different positions, and

\section{POSITION AVAILABLE Electron Tomography/ Image Processing}

The Wadsworth Center seeks a motivated, responsible and mature individual to develop and implement procedures in electron tomography for federally-funded biomedical research projects. The electron microscopy facility at the Wadsworth Center is state of the art with an F20 and F30 FEI Tecnai microscopes, a JOEL 4000 with energy filter, and Zeiss 910 , all with capability for automated data collection for electron tomography. The Wadsworth Center also has an AEI 1.2 MV HVEM for thick section work, and a state of the art light microscopy facility for correlative studies. The research environment is stimulating and challenging and includes several well-funded Pl's in cell and structural biology. Training will be provided for all specialized tasks.

Minimum qualifications: B.S. in physics, computer science, mathematics, chemistry, or biology; computer competence and familiarity with $\mathrm{PC}$ and Unix operating system. Preferred Qualifications: B.S. in physics or computer science; experience with transmission electron microscopy; experience with image processing; programming capability.

Responsibilities: Compute and analyze tomographic reconstructions for grant-funded projects; assist in developing and implementing new procedures for automating tomographic reconstruction and volume segmentation; implement and manage a database cataloging input data, project parameters, critical procedures; results of the $3 \mathrm{D}$ reconstruction and the $3 \mathrm{D}$ analysis.

Salary commensurate with experience. Interested parties should contact:

Dr. Bruce F. McEwen

c/o Ms. Cathy Kahler

Wadsworth Center

PO Box 509

Albany, NY 12201-0509

Tel: (518) 4862882 Fax (518) 4864901

Email: ckahler@wadsworth.org 